\title{
A PRELIMINARY ANALYSIS OF PHOTOELECTRIC OCCULTATION MEASUREMENTS
}

\author{
E. PANSCH and CHR. DE VEGT
}

Hamburger Sternwarte, 2050 Hamburg, B.R.D.

\begin{abstract}
Photoelectric occultation traces of 5 stars are discussed which have been obtained with the $60 \mathrm{~cm}$ refractor of the Hamburg-Observatory during 1969/70. All traces show remarkable deviations from the usual Fresnel point-source pattern. Most details of these traces can be interpreted assuming the stars to be unresolved doubles. A short description of the equipment and the observational procedure is given.
\end{abstract}

\section{Introduction}

Photoelectric observations of lunar occultations as a routine program have been started at the Hamburg-Observatory in April 1969 with the $60 \mathrm{~cm} f: 15$ refractor.

The photoelectric equipment used consists of a pulse-counting photometer, an on-line PDP8/S small computer for data storing and processing and a quartz controlled digital clock with separate display-memory for exact timing down to $1 \mathrm{msec}$. A detailed description of the photometer, the electronics and the operational mode can be found in Schlosser and de Vegt (1968), Schlosser et al., (1970), Hög (1970). A general block-diagram of the equipment is shown in Figure 1.

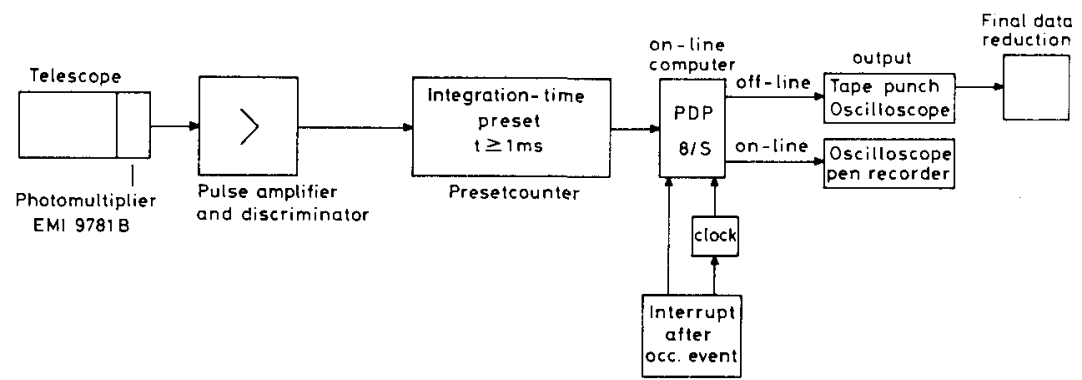

Fig. 1. Block-diagram of the observational equipment.

Due to the relatively small size of the telescope the resolution of an occultation curve with an integration-time of $1 \mathrm{msec}$ is only possible for stars not fainter than $m_{v}=8$, provided the amount of scattered moonlight can be neglected. Under unfavourable observing conditions this limiting magnitude may be decreased by 2-3 mag. In addition, timing of the event is possible down to $m_{v}=9$ with an accuracy of a few milliseconds (Pansch and de Vegt, 1970).

A specific difficulty in occultation work may arise from the observation of reappearance events, as most telescopes are not prepared to be used with an offsetguiding technique. In our case offset-guiding is achieved by a special arrangement of the photometer-head. It is mounted on a groundplate which contains a slit with a 
second guiding-ocular, which can be shifted by a motor-drive along a radius vector, the origin of which is the photometer diaphragm, situated in the optical axis of the telescope. In addition the whole photometer-groundplate can be rotated around the optical axis, so that the celestial coordinates of the diaphragm can be adjusted to a prescribed position with an accuracy of 1-2 sec of arc.

During an occultation measurement the counted photons are continuously stored in a core memory loop containing 3315 consecutive locations. This corresponds to a time interval of $3.315 \mathrm{sec}$, when an integration time of $1 \mathrm{msec}$ is used. After the occultation event has occurred, the observer has to release the interrupt which stops the cyclic storing process in the memory-loop and retains the interrupt time $t_{i}$ in the display-memory of the digital clock (Figure 1).

Those measuring data are stored, being inside the interval $t_{i}>t>t_{i}-3.315 \mathrm{sec}$. So the interrupt has to be released not later than about $t+2 \mathrm{sec}$ otherwise that part of the memory-loop containing the occultation curve will be destroyed. During the storing process, the measured intensity is simultaneously plotted on the oscilloscope, so that the observer can recognize the intensity-jump due to the occultation event. This method could be of great advantage in the case of faint stars or large amounts of scattered moonlight where the event cannot be seen at the guiding-telescope.

Attempts to detect the occultation automatically when the intensity passes through a certain level were not satisfactory due to rapidly changing amounts of scattered light.

\section{Discussion of Observations}

A preliminary analysis of 6 occultation traces from 5 different stars has been made, assuming that the observed pattern is the superposition of two point-source stars. Theoretical occultation curves were computed, taking in account the spectral response of the multiplier + filter combination and the spectral type of the star, if known.

The parameters, to be varied to give the best representation of the observed occultation curve are:

the intensity-ratio of the components $\gamma$;

the distance of the components in the direction of lunar motion $\tau$ [msec];

the timescale of the event;

the occultation-time for one component.

From these data, only the vector-separation $\tau_{v}$ of the components can be computed if no further observation at a different limb position is available.

Most of the traces have been obtained under unfavourable observing conditions. Due to the low altitude of the observatory and its site near Hamburg, a considerable amount of scattered moonlight is mostly present.

$$
19 \mathrm{TAU}=\mathrm{BD}+24^{\circ} 547 \text {. }
$$

Date of observation: 30.9.69; reappearance event; EMI + BG12, $2 \mathrm{~mm}$. The star has been supposed to be a spectroscopic double. (Abt et al., 1965). A further observation has been made by Elliott (Elliott et al., 1970). 


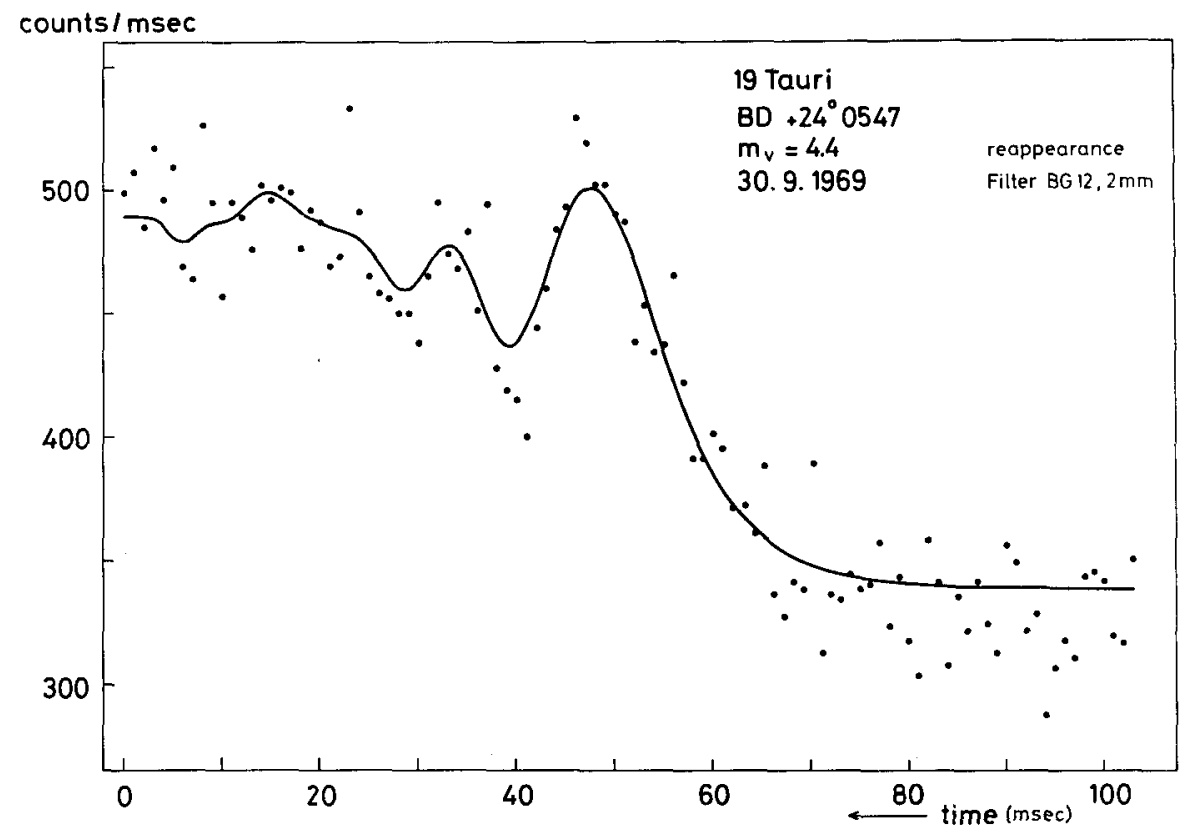

Fig. 2.

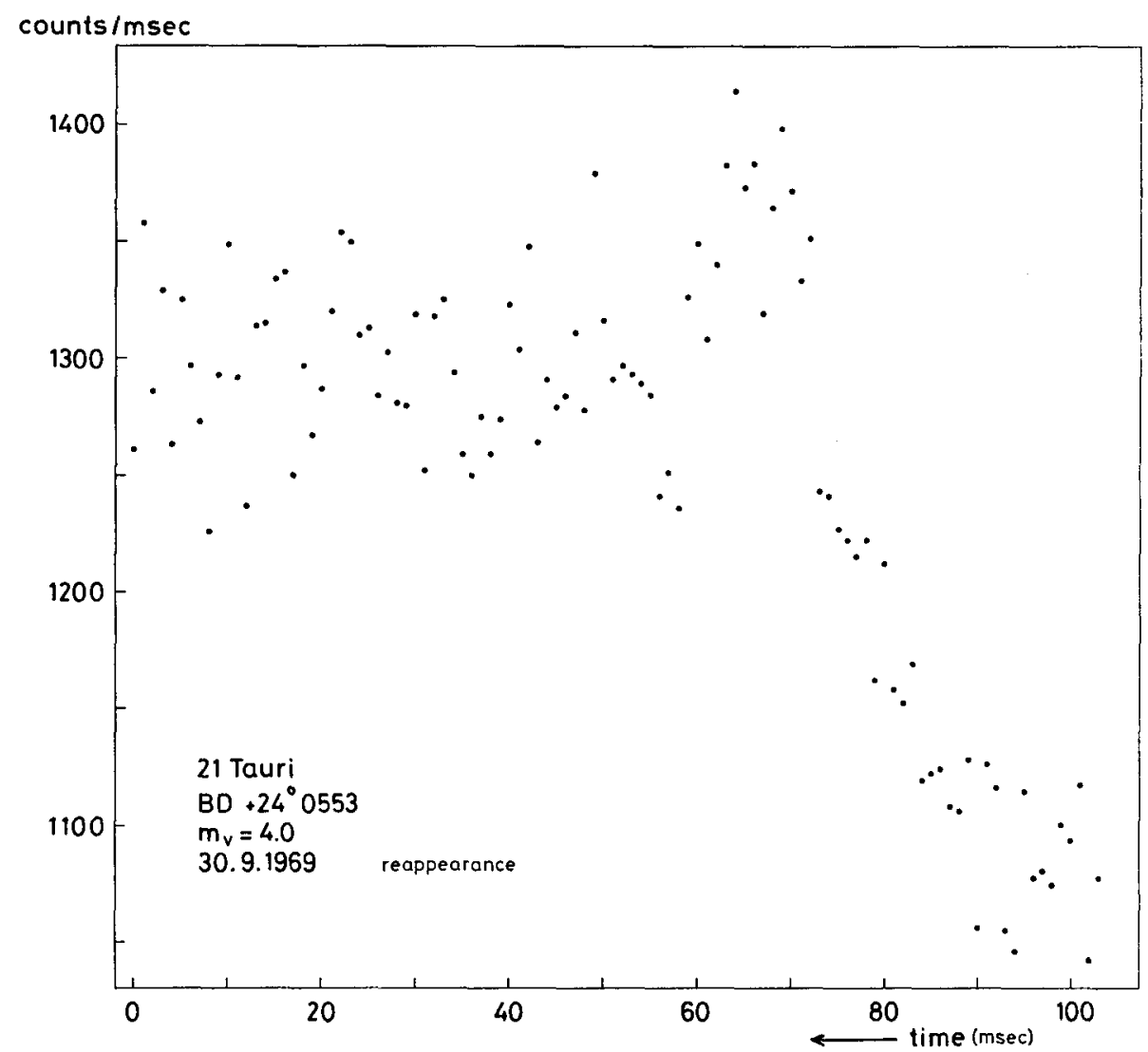




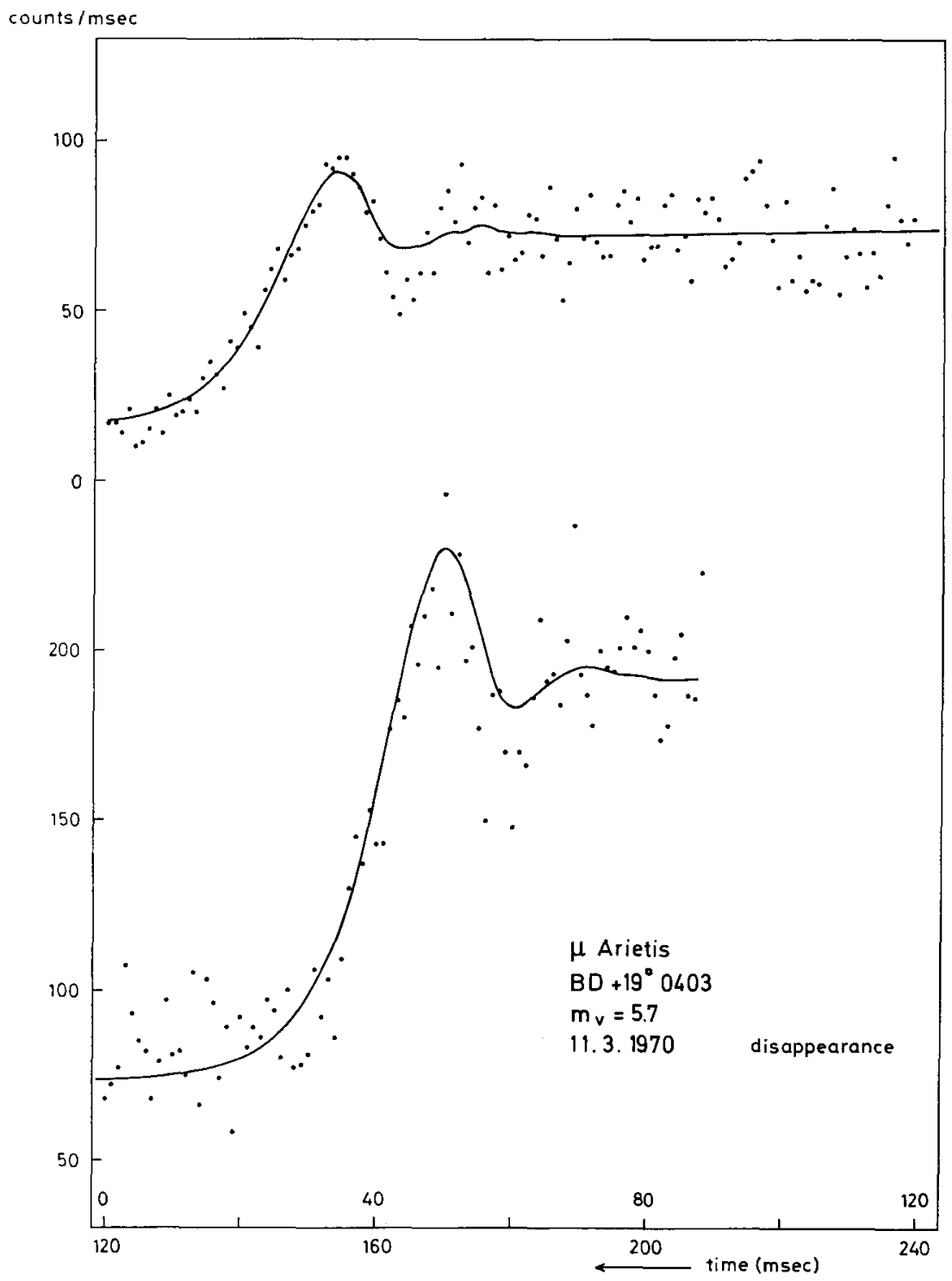

Fig. 4.

\section{Computed parameters:}

Intensity-ratio $\gamma=0.245$

vector-separation $\tau_{v}=0 " 012$

Amount of scattered light: $230 \%$ (Intensity of star $=1$ ). 


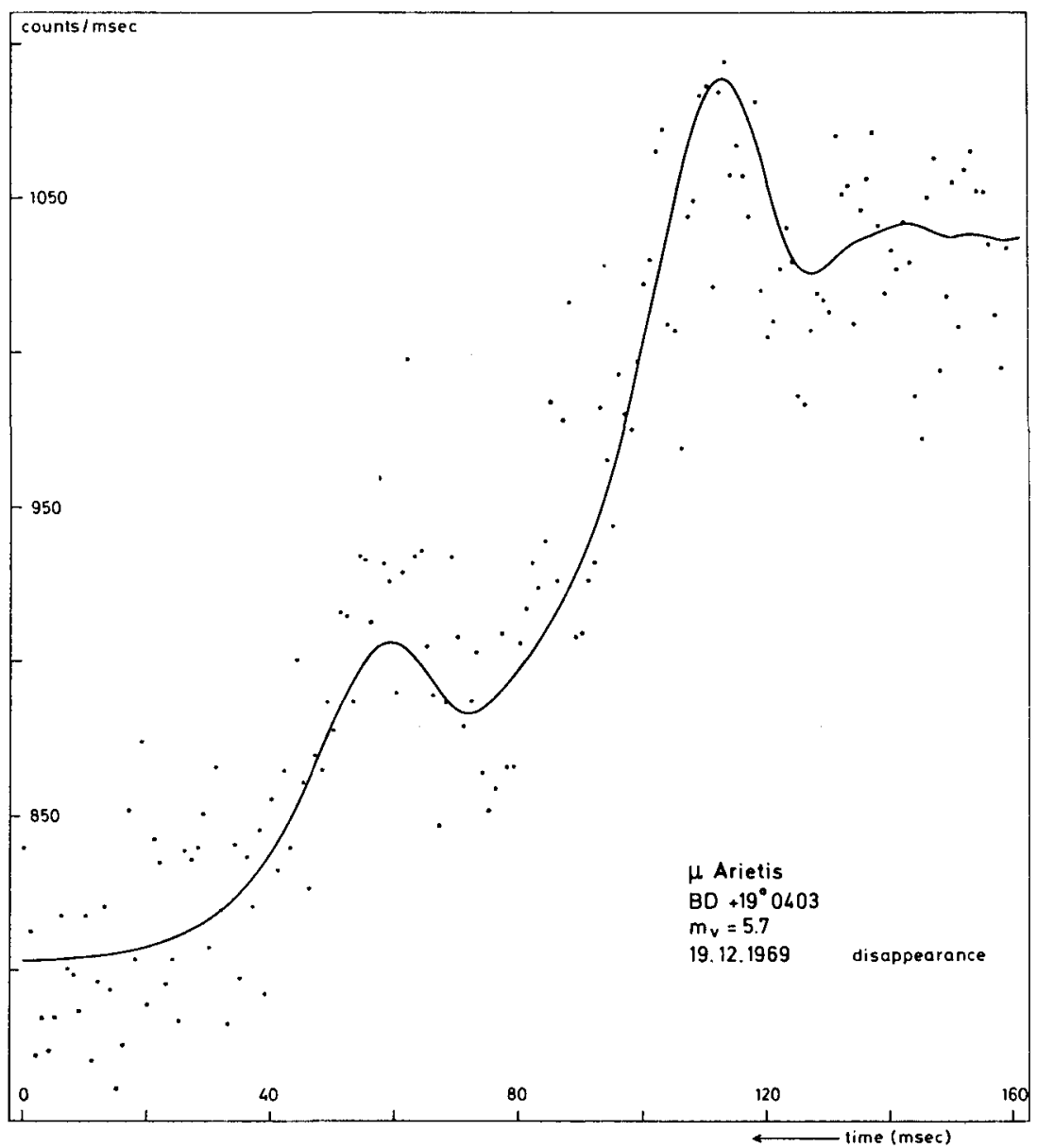

Fig. 5.

$$
21 \mathrm{TAU}=\mathrm{BD}+24^{\circ} 553 .
$$

Date of observation: 30.9.69; reappearance event; EMI. According to the recent list of Herr (1969), the star does not seem to be known as a double. The observed occultation trace is quite peculiar and may be disturbed by limb irregularities. The observation could not be represented by a theoretical occultation trace. A second observation (21.12.69) is available from Elliott et al. (1970). According to these authors the star seems to be a double.

In our case the amount of scattered light reached $400 \%$.

$$
\mu \mathrm{ARI}=\mathrm{BD}+19^{\circ} 403 .
$$

Date of observation: 19.12.69; disappearance event; EMI. This star itself is a visual binary, the bright component (ADS 2062A) is not included in Herr's list and seems to be an unexpected double. 


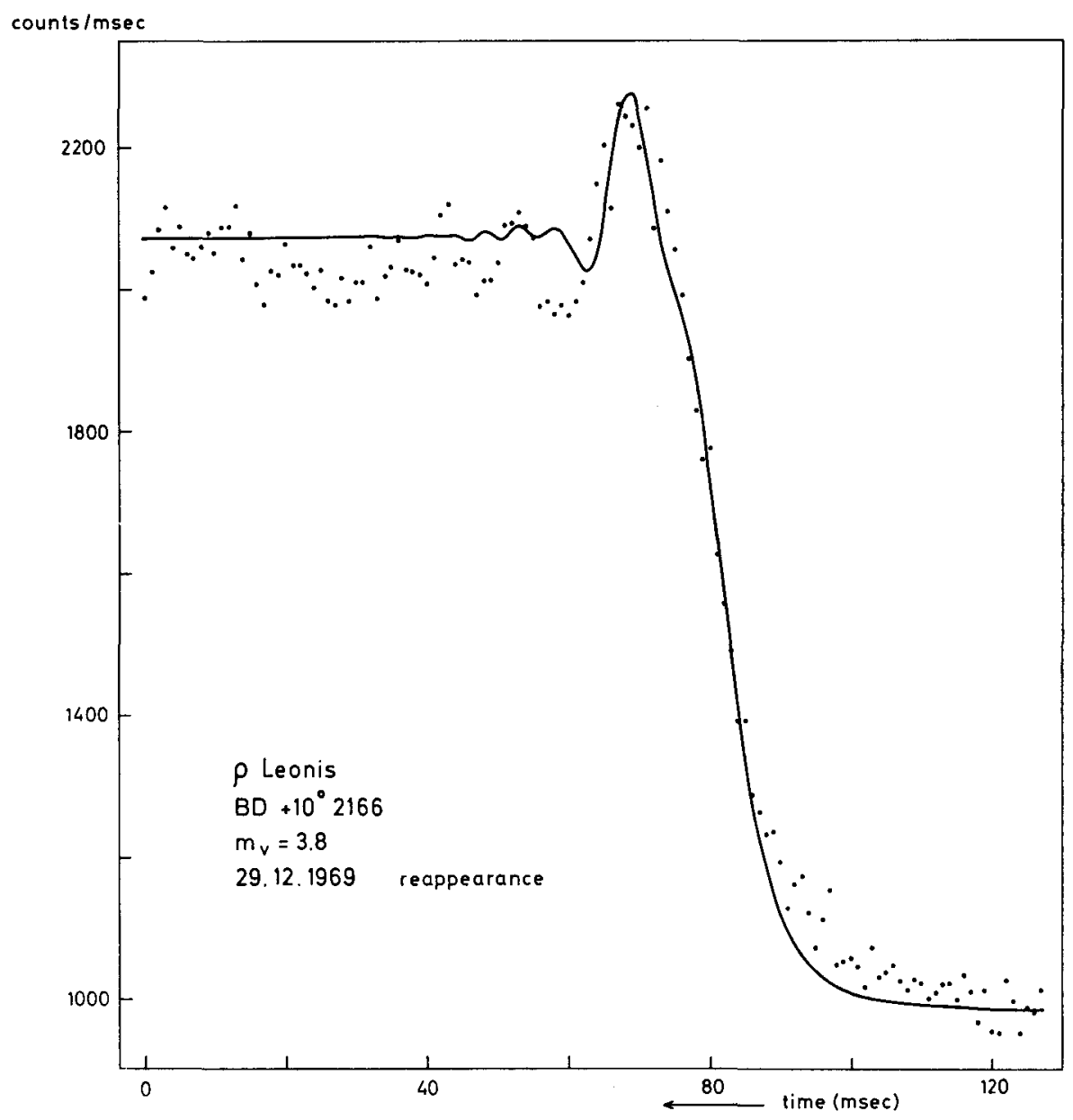

Fig. 6 .

\section{Computed parameters:}

Intensity-ratio $\gamma=2.10$

vector-separation $\tau_{v}=0$ ".013

Amount of scattered light: $338 \%$.

$$
\mu \mathrm{ARI}=\mathrm{BD}+19^{\circ} 403 .
$$

Date of observation: 11.3.70; disappearance event; EMI.

Computed parameters:

Intensity-ratio: $\gamma=2.10$

vector-separation $\tau_{v}=0$ ".042

Amount of scattered light: $9 \%$ 
From the observations 3.1 and 3.2 the distance and position angle of the components has been estimated: $\varrho=0.057 ; p=83^{\circ}$.

$$
\varrho \mathrm{LEO}=\mathrm{BD}+10^{\circ} 2166 .
$$

Date of observation: 29.12.69; reappearance event; EMI. The star is known as a spectroscopic double, orbital elements are not available. (Batten, 1967; Herr, 1969).

Computed parameters:

Intensity-ratio $\gamma=1.45$

vector-separation $\tau_{v}=0^{\prime \prime} .0036$

Amount of scattered light: $90 \%$

$$
\mathrm{BD}+24^{\circ} 1805 \text {. }
$$

Date of observation: 13.4.70; disappearance event; EMI

The star seems to be an unexpected double.

Computed parameters:

Intensity-ratio: $=0.512$

vector-separation $=0.0874$

Amount of scattered light: $116 \%$.

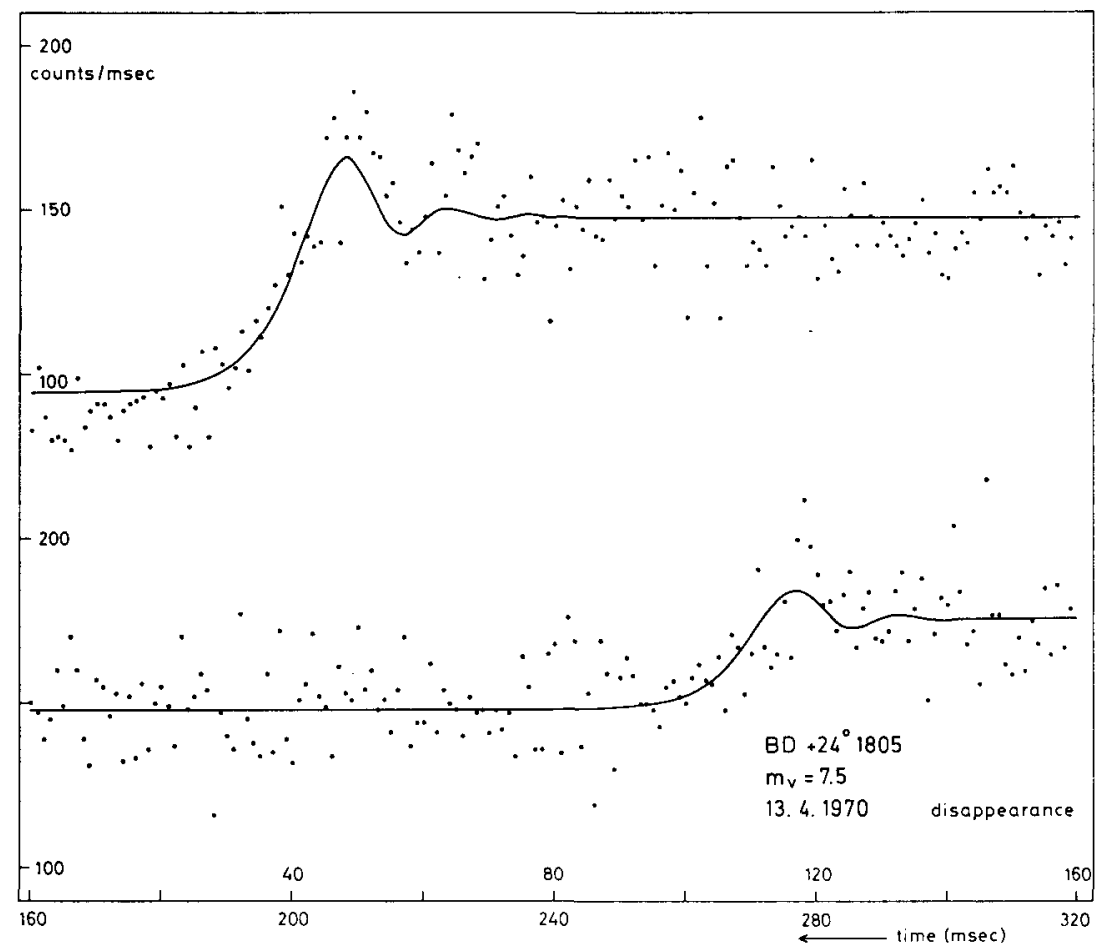

Fig. 7. 


\section{Future Observing Program}

This occultation program will be continued at the Hamburg-Observatory with the equipment described above. In co-operation with Dr K. Rakos, Vienna, we shall also have the opportunity to use the new Austrian $1.5 \mathrm{~m}$ telescope. As our present counting system - developed by E. Høg for his scanning microphotometer - has a bandwidth of $10 \mathrm{Mc}$, it is not suitable to be used for occultation measurements with a considerable larger telescope. To have maximum information from a single occultation event we have a three-channel photometer under construction now, containing an EMI 9781 B multiplier, a RCA 4526 and - following a suggestion by K. Rakos - a PINphotodiode HP 4204 for the blue, yellow and red spectral range respectively. For colour-splitting, Dichroic filters will be used. According to the spectral type of the star at least 2 photometer-channels will be used simultaneously. Amplification and analog-digital conversion will be achieved by commercially available nuclear research equipment. For data storing and processing an on-line Hewlett-Packard small computer HP $2115 \mathrm{~A}$ is planned.

\section{Acknowledgements}

The research was supported by the Deutsche Forschungsgemeinschaft (German Science Foundation) in Bad Godesberg.

\section{References}

Abt, H. A., Barnes, R. C., Biggs, E. S., and Osmer, P. S.: 1965, Astrophys. J. 142, 1604.

Batten, A. H.: 1967, Publ. Dom. Astrophys. Obs. 13, 119.

Elliott, J. E., Evans, D. S., Nather, R. E., and Wild, P. A. T.: 1970, Astron. J. (in press).

Herr, R. B.: 1969, Publ. Astron. Soc. Pac. 81, 105.

Hög, E.: 1970, 'A Data Aquisition System with On-Line Computer' (to be publ. in Highlights of Astronomy 1970, IAU XIV.

Pansch, E. and de Vegt, Chr.: 1970, Astron. Astrophys. 5, 328-329.

Schlosser, W. and de Vegt, Chr.: 1968, 'Bericht über ein Sternbedeckungsphotometer' in Mitt. Astron. Gesellschaft 25, p. 173.

Schlosser, W., Pansch, E., and de Vegt, Chr.: 1970, Astron. Astrophys. (in press).

\section{DISCUSSION}

David W. Dunham: I was particularly interested in your results for $\mu$ Arietis because I observed a grazing occultation of this star on 17 January, 1970. Five disappearances and five reappearances of the star occurred. Three or four of these events were noticeably slow, requiring up to a second. Once the star dimmed, but did not completely disappear, then regained full brilliance, in a period of $2 \frac{1}{2} \mathrm{sec}$. Yet from Pansch's results, the vector separation must have been only about $0 .{ }^{\prime \prime} 02$ for the graze. This demonstrates the utility of grazing occultation observation for detecting close binaries. 